\title{
Three-dimensional analysis reveals two major architectural subgroups of prostate cancer growth patterns
}

\author{
Esther I. Verhoef $\mathbb{B}^{1} \cdot$ Wiggert A. van Cappellen ${ }^{1,2} \cdot$ Johan A. Slotman $\mathbb{1}^{1,2} \cdot$ Gert-Jan Kremers ${ }^{1,2} \cdot$ \\ Patricia C. Ewing-Graham ${ }^{1}$. Adriaan B. Houtsmuller ${ }^{1,2} \cdot$ Martin E. van Royen $^{1,2} \cdot$ Geert J. L. H. van Leenders ${ }^{1}$
}

Received: 22 October 2018 / Revised: 16 January 2019 / Accepted: 17 January 2019 / Published online: 8 February 2019

(c) The Author(s) 2019. This article is published with open access

\begin{abstract}
The Gleason score is one of the most important parameters for therapeutic decision-making in prostate cancer patients. Gleason growth patterns are defined by their histological features on 4- to 5- $\mu \mathrm{m}$ cross sections, and little is known about their threedimensional architecture. Our objective was to characterize the three-dimensional architecture of prostate cancer growth patterns. Intact tissue punches $(n=46)$ of representative Gleason growth patterns from radical prostatectomy specimens were fluorescently stained with antibodies targeting Keratin 8/18 and Keratin 5 for the detection of luminal and basal epithelial cells, respectively. Punches were optically cleared in benzyl alcohol-benzyl benzoate and imaged using a confocal laser scanning microscope up to a depth of $500 \mu \mathrm{m}$. Gleason pattern 3, poorly formed pattern 4, and cords pattern 5 all formed a continuum of interconnecting tubules in which the diameter of the structures and the lumen size decreased with higher grades. In fused pattern 4 , the interconnections between the tubules were markedly closer together. In these patterns, all tumor cells were in direct contact with the surrounding stroma. In contrast, cribriform Gleason pattern 4 and solid pattern 5 demonstrated a three-dimensional continuum of contiguous tumor cells, in which the vast majority of cells had no contact with the surrounding stroma. Transitions between cribriform pattern 4 and solid pattern 5 were seen. There was a decrease in the number and size of intercellular lumens from cribriform to solid growth pattern. Glomeruloid pattern 4 formed an intermediate structure consisting of a tubular network with intraluminal epithelial protrusions close to the tubule splitting points. In conclusion, three-dimensional microscopy revealed two major architectural subgroups of prostate cancer growth patterns: (1) a tubular interconnecting network including Gleason pattern 3, poorly formed and fused Gleason pattern 4, and cords Gleason pattern 5, and (2) serpentine contiguous epithelial proliferations including cribriform Gleason pattern 4 and solid Gleason pattern 5.
\end{abstract}

\section{Introduction}

The Gleason score is one of the most important parameters for therapeutic decision-making in men with prostate cancer and is entirely based on tumor growth patterns $[1,2]$. Tumor heterogeneity is recognized by adding the two most common

Supplementary information The online version of this article (https:// doi.org/10.1038/s41379-019-0221-0) contains supplementary material, which is available to authorized users.

Esther I. Verhoef

e.verhoef@erasmusmc.nl

1 Department of Pathology, University Medical Center Rotterdam, Rotterdam, The Netherlands

2 Department of Optical Imaging Center, Erasmus MC, University Medical Center Rotterdam, Rotterdam, The Netherlands
Gleason patterns in radical prostatectomy specimens. Gleason pattern 1,2, and 3 prostate cancers are composed of welldelineated malignant glands, and the distinction of these patterns is putatively of no clinical significance [3]. Gleason pattern 4 tumors consist of poorly formed, fused, cribriform, or glomeruloid structures. Tumor growth in cords, single cells or solid fields, or the presence of comedonecrosis, characterizes Gleason pattern 5. Whereas men with Gleason score 6 (ISUP group 1) prostate cancer are often eligible for surveillance, active treatment is usually offered to patients with Gleason score $\geq 7$ (ISUP group $\geq 2$ ) [4].

Although individual growth patterns within Gleason patterns 4 and 5 are not routinely mentioned in pathology reports, numerous studies have demonstrated poorer outcomes when cribriform growth is present [5-9]. Cribriform growth in radical prostatectomies and diagnostic biopsies has been associated with more post-operative biochemical recurrence and disease-specific death in International 
Society of Urological Pathology (ISUP) group $\geq 2$ prostate cancer patients [7]. On the other hand, ISUP group 2 patients with glomeruloid architecture may have a better outcome than those without this pattern [8]. Consideration of individual growth patterns may therefore have added value in the therapeutic stratification of ISUP group 2 prostate cancer patients.

A major limitation of the Gleason grading system is the substantial inter-observer variability [10-12]. Egevad et al. found that in a group of 337 European pathologists, only $56 \%$ agreement was achieved between expert consensus and participants' score [13]. Inter-observer variability in Gleason grading occurs predominantly in the assessment of poorly formed and fused growth patterns [5, 7, 8, 12, 14]. In particular, small glands with sporadic lumen formation may be interpreted as tangentially sectioned Gleason pattern 3, poorly formed pattern 4 , or cords pattern 5 . Inter-observer variability significantly affects clinical decision-making since the distinction of ISUP group 1 prostate cancer from higher grades is an important threshold for active surveillance and treatment [4].

Diagnostic criteria for the histopathological grading of prostate cancer are entirely based on tumor features of routine 4- to $5-\mu \mathrm{m}$ tissue sections. Very little is known about the underlying three-dimensional architecture of Gleason growth patterns. Serial sectioning and scanning of many tissue sections have given some insight into the three-dimensional tumor architecture; however, this is costly, time-consuming, and susceptible to artifacts. In contrast, optical tissue clearing allows for the sensitive fluorescent imaging of whole-tissue specimens without physical sectioning [15-18]. We have already demonstrated the feasibility of this technique for three-dimensional visualization of formalin-fixed, paraffinembedded prostate tissues up to a depth of $800 \mu \mathrm{m}$ [19]. The objective of the current study was to characterize and provide a comprehensive overview of the three-dimensional architecture of prostate cancer growth patterns.

\section{Materials and methods}

\section{Case selection}

Archival formalin-fixed, paraffin-embedded radical prostatectomy specimens from patients who had undergone radical prostatectomy for prostate cancer at the Erasmus Medical Center between 2012 and 2017 were included. Specimens were fixed in neutral-buffered formalin, transversely cut into 4-mm slices, and entirely embedded for histopathologic evaluation. The mean age at operation was 66 years (SD 6.8 years). Regions of interest for threedimensional imaging were indicated on hematoxylin and eosin-stained slides by a urogenital pathologist. In total, 46 tumor areas from 35 patients were selected for analysis, including Gleason pattern $3(n=8)$, poorly formed $(n=6)$, fused $(n=6)$, glomeruloid $(n=10)$ and cribriform $(n=6)$ Gleason pattern 4 , and cords $(n=7)$ and solid-fields $(n=3)$ Gleason pattern 5 . Three normal peripheral zone areas were included to serve as a reference. The use of tissue samples for scientific purposes was approved by the institutional Medical Research Ethics Committee (MEC-2011-295, MEC-2011-296) and was in accordance with the "Code for Proper Secondary Use of Human Tissue in The Netherlands" as developed by the Dutch Federation of Medical Scientific Societies (FMWV, version 2002, update 2011).

\section{Immunofluorescent staining and optical clearing}

Tissue punches from the areas with the selected growth patterns were taken from the corresponding paraffin blocks using a 500- $\mu \mathrm{m}$ diameter needle (Estigen Tissue Scuebcem, Tartu, Estonia) resulting in 3- to 4-mm-long cylindrical tissue cores with a diameter of $500 \mu \mathrm{m}$. Immunofluorescent staining and optical clearing were carried out according to an adapted iDISCO protocol as described previously (Supplementary table 1) [19, 20]. Briefly, punches were dewaxed, after which auto-fluorescence was blocked overnight. Subsequently, the punches were gradually rehydrated and incubated with primary Keratin 5 and Keratin 8/18 antibodies (1:150; EP1601Y; Abcam, Cambridge, UK and 1:75; MS-743; Immunologic, Duiven, The Netherlands) and secondary fluorescent Alexa-514- and Alexa-647-labeled antibodies (1:200; Life Technologies, Bleiswijk, The Netherlands). In order to visualize the subtle connective tissue cores within fused Gleason pattern 4, these samples were additionally stained with Fibronectin (FN1; 1:50; ab2413; Abcam, Cambridge, United Kingdom) and secondary Alexa-647 antibodies. The tissue was again dehydrated in methanol and subsequently optically cleared in benzyl alcohol-benzyl benzoate. Samples were then stored at $4{ }^{\circ} \mathrm{C}$ in the dark until imaging.

\section{Sample imaging}

Fluorescently stained punches were imaged with an upright Leica SP5 confocal microscope equipped with a $1.95-\mathrm{mm}$ working distance $20 \times$ NA1.0 APO water dipping objective (Leica Microsystems GmbH, Wetzlar, Germany). Twodimensional $Z$-stack images were recorded using a $488 \mathrm{~nm}$ Argon and a $633 \mathrm{~nm}$ HeNe laser with a $0.72 \times 0.72 \mu \mathrm{m}$ pixel size and 1-3 $\mu \mathrm{m}$ step size, resulting in 300-600 images per sample. Huygens Professional software (SVI, Hilversum, The Netherlands) with a theoretical point-spread function was used for de-convolution of the $Z$ stacks, whereas three-dimensional rendering and image measurements were performed with Fiji (ImageJ 1.49s) and Amira (version 5.5.0; ThermoFisher 
Scientific, Waltham, USA) software [21]. Z stacks were loaded in Amira, after which we applied combined surface and volume rendering with standard settings. The total size of the $Z$ stacks and three-dimensional renderings was 739 by $739 \mu \mathrm{m}$ with a depth of $500 \mu \mathrm{m}$. Reference hematoxylin and eosin slides were positioned at a vertical side of the threedimensional renderings, but were not directly continuous with the depicted areas in each case, depending on the site of imaging in the 3- to 4-mm-long cylindrical core.

\section{Pathological evaluation and statistical analysis}

Both consecutive $Z$ stacks and three-dimensionally rendered images were investigated. Pre-existent benign prostate glandular structures were identified by Keratin 8/18-positive luminal cells surrounded by Keratin 5-positive basal cells. Prostate cancer structures were recognized by architecturally disorganized Keratin 8/18-positive epithelial structures without a basal cell layer. Tubular blind-ending tips were identified by a detailed analysis of both $Z$ stacks and three-dimensional renderings, and could be well distinguished from the transversely sectioned tubules at the border of the tissue samples. In each of the tissue specimens, the outer edges of the epithelial structures were measured in 3-5 consecutive two-dimensional slides per image and 3-10 individual epithelial structures per slide, depending on the growth pattern. This resulted in 12-50 measurements per tissue sample. Statistics were performed with a Student's $t$ test using the Statistical Package for Social Sciences (SPSS, version 24; IBM, Chicago, USA).

\section{Results}

\section{Benign epithelial glands}

Benign peripheral zone glands had an acinar organization composed of interconnecting saccules with variable intraluminal papillary protrusions. Benign glands were composed of an inner Keratin 8/18-positive luminal cell layer and an outer flat Keratin 5-positive layer of basal cells (Fig. 1a, b; Supplementary Video 1). In all cases, malignant epithelial structures could easily be distinguished from preexistent benign glands by their architectural organization and lack of basal cells (Fig. 1c, d).

\section{Gleason pattern 3}

Gleason pattern 3 prostate cancer was composed of round to slightly oval, well-delineated curving tubules with a mean diameter of $45 \mu \mathrm{m}$ (SD $12 \mu \mathrm{m})$ with regular interconnections (Fig. 2a, b; Supplementary Video 2). All malignant epithelial cells had contact with surrounding stroma. We did not find any specific tubular orientation along the cranial-caudal, transverse, or sagittal axis of the prostate. Blind-ending tubules were present sporadically and showed no specific location within the tubular network.

\section{Gleason pattern 4}

\section{Poorly formed glands}

Poorly formed Gleason pattern 4 glands were represented by small round tubules with a significantly smaller average diameter than Gleason pattern 3 tubules $(24 \mu \mathrm{m}, \mathrm{SD} 7 \mu \mathrm{m}$; $p<0.001)$. Poorly formed glands showed more frequent interconnections and blind endings than Gleason pattern 3 tubules (Fig. 2c, d). Regularly, we observed transitions between poorly formed Gleason pattern 4 glands and Gleason pattern 3 tubules (Fig. 2e; Supplementary Video 3).

\section{Fused glands}

Fused Gleason pattern 4 glands consisted of round to oval tubules with a diameter of $68 \mu \mathrm{m}$ (SD $18 \mu \mathrm{m}$ ), slightly larger than that of Gleason pattern 3 tubules $(p<0.001)$. The hallmark of the fused pattern was the presence of abundant interconnections between the tubules, which occurred markedly closer together than in the aforementioned patterns (Fig. 3a, b). On hematoxylin and eosin slides, fused Gleason pattern 4 can closely resemble the cribriform architecture [12, 13]. Here three-dimensional microscopy, however, revealed subtle intervening fibrovascular tissues in between and around all malignant tubules, revealing that all malignant cells had contact with the surrounding stroma (Supplementary Video 4).

\section{Cribriform fields}

Cribriform Gleason pattern 4 was characterized by fields of contiguous epithelial tumor cells with a mean diameter of $151 \mu \mathrm{m}$ (SD $68 \mu \mathrm{m}$ ). The vast majority of tumor cells did not have any contact with the surrounding stroma, in contrast with the patterns described previously. Three dimensionally, this pattern showed a variable number of spherical, ellipsoid, slit-like, or irregular interconnecting intercellular lumens (Fig. 3c, d). Adjacent cribriform fields with intervening stroma on hematoxylin and eosin slides represented continuously curving irregular serpentine structures on three-dimensional renderings (Supplementary Video 5). We did not observe any transition between cribriform fields and aforementioned tubular structures in our cohort.

\section{Glomeruloid glands}

In two-dimensional cross sections, glomeruloid Gleason pattern 4 structures resemble renal glomeruli and are 
Fig. 1 Peripheral zone a hematoxylin and eosin slide and b three-dimensional rendering, showing interconnecting saccules of variable width containing intraluminal papillary protrusions and surrounded by a continuous Keratin 5-positive basal cell layer (red).

c Hematoxylin and eosin slide and $\mathbf{d}$ three-dimensional rendering of pre-existent benign glands surrounded by basal cells (upper left) and irregular malignant epithelial structures without a basal cell layer (lower). Original magnifications $20 \times$; green, Keratin 8/18 and red, Keratin 5 immunostaining in three-dimensional renderings
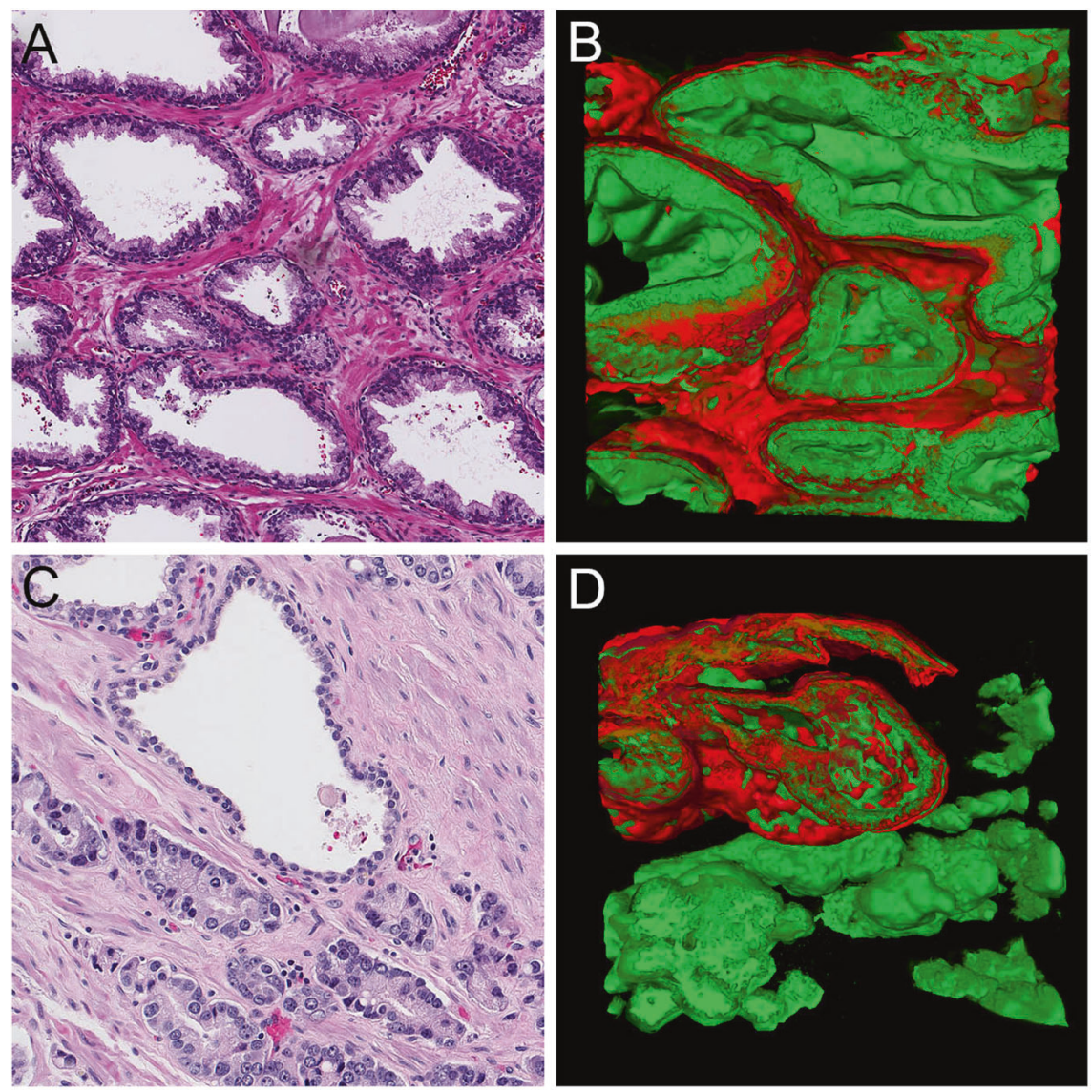

characterized by dilated glands with round protrusions of malignant epithelial cells (Fig. 4a, b). On threedimensional renderings, these glomeruloid structures were present within an interconnecting network of tubules, which had a mean diameter of $65 \mu \mathrm{m}$ (SD $19 \mu \mathrm{m})$, reminiscent of Gleason pattern 3 glands, but with larger tubule diameters $(p<0.001)$. Two different glomeruloid structures could be distinguished using three-dimensional microscopy. The first type was nodular epithelial glomeruloid proliferations, which connected to the tumor cells lining the tubule on one side, but did not make any contact with the tubular lining on the opposite side or the surrounding stroma. These protrusions often occurred at tubular branching points (Supplementary Video 6). The second type showed the presence of subtle fibrovascular cores on the hematoxylin and eosin slides, representing clusters of markedly curved tubules in three dimensions. All the tumor cells within this glomeruloid variant made contact with the surrounding stroma. When glomeruloid structures are larger, distinction between glomeruloid and cribriform growth patterns on hematoxylin and eosin slides can be challenging (Fig. 4c) [12]. These larger cribriform-like structures grew similar to the first type of glomeruloid pattern in three-dimensional renderings. We did not observe any continuity between glomeruloid structures and the cribriform pattern.

\section{Gleason pattern 5}

\section{Cords and single cells}

On hematoxylin and eosin slides, Gleason pattern 5 cords consist of one- or two-layered strands of cells without distinctive lumens (Fig. 5a). In three dimensions, cords and single-cell structures formed a continuous meshwork consisting of one or two cell layers with extensive branching and interconnections. The average diameter of these cords was $15 \mu \mathrm{m}$ (SD $7 \mu \mathrm{m}$; Fig. 5b), significantly smaller than poorly formed Gleason pattern $4(p<0.001)$. Small intercellular lumens were observed at deeper levels of the cord pattern in the $Z$ stack, indicating repetitive subtle transitions from Gleason pattern 4 poorly formed tubules to Gleason pattern 5 cords (Supplementary Video 7).

\section{Solid fields}

Solid-fields Gleason pattern 5 are represented on hematoxylin and eosin slides as round or irregularly formed 
Fig. 2 Gleason pattern 3 a hematoxylin and eosin slide and b three-dimensional rendering showing a tubular network with interconnections (arrows) and blind endings (arrowheads). Poorly formed Gleason pattern 4 c hematoxylin and eosin slide and $\mathbf{d}$ three-dimensional rendering of small-sized interconnecting tubules with blind endings (arrowheads). e Well-delineated Gleason pattern 3 tubules (arrows) were directly connected to and were continuous with poorly formed Gleason pattern 4 structures (arrowheads). Original magnifications $20 \times$; green, Keratin 8/18 and red, Keratin 5 (no basal cells present) immunostaining in threedimensional renderings
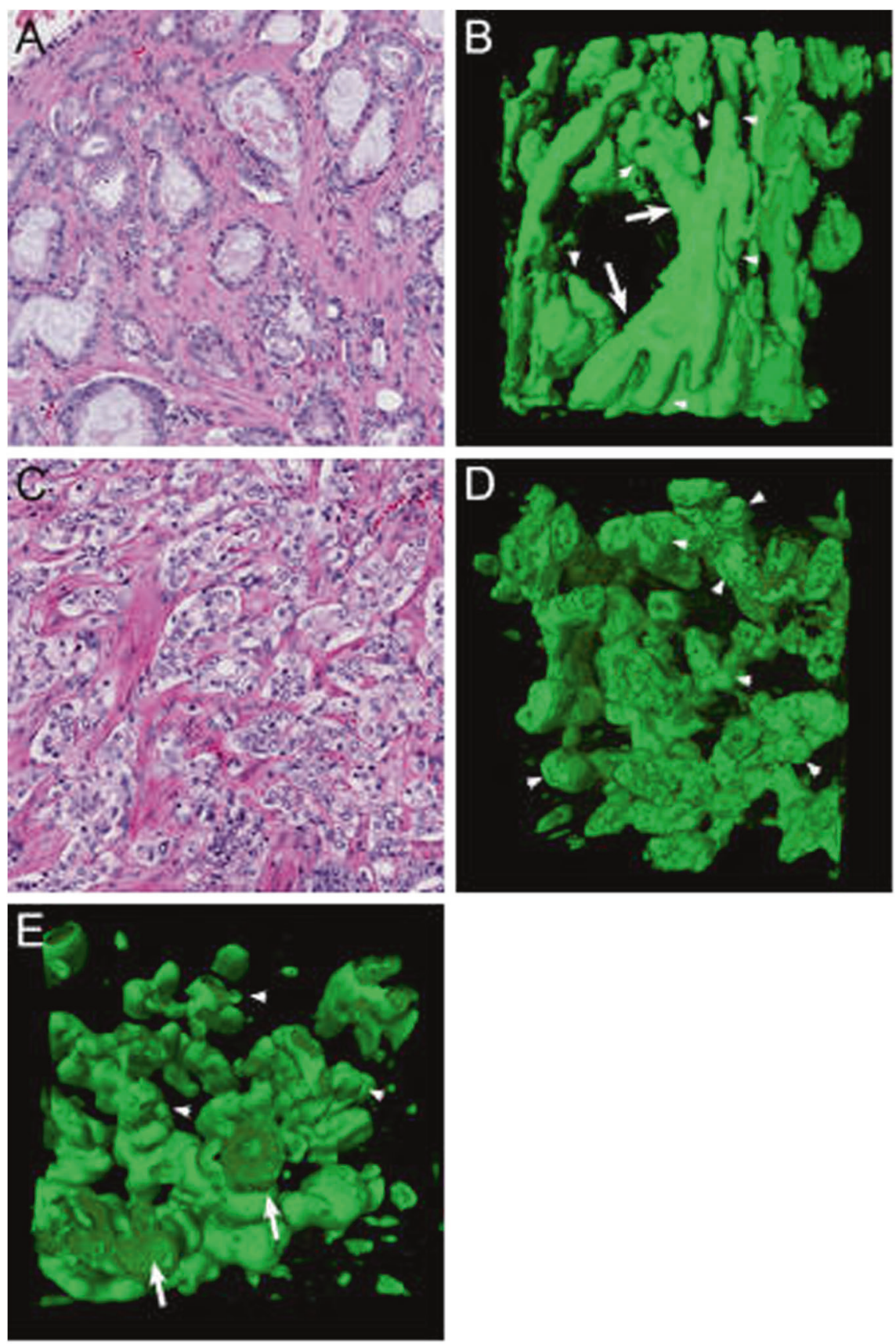

areas composed of tumor cells without intercellular lumens. Most cells do not make any contact with the surrounding stroma (Fig. 5c). Three-dimensional reconstruction has revealed that the solid fields represented round to ellipsoid irregular serpentine structures with an average diameter of $185 \mu \mathrm{m}$ (SD $78 \mu \mathrm{m}$ ), interconnecting and varying in width (Fig. 5d; Supplementary Video 8). While intercellular lumens were inconspicuous on hematoxylin and eosin slides, the $Z$ stacks often showed small, round, and ellipsoid lumens, reminiscent of cribriform Gleason pattern 4 at deeper levels, indicating a transition between these patterns. In this cohort, we did not find any transition between solid-fields Gleason pattern 5 and tubular growth patterns.

\section{Discussion}

In the current study, we provided a comprehensive overview of the three-dimensional architecture of prostate cancer growth patterns and revealed two architecturally different growth pattern subgroups. The first subgroup consisted of a tubular network in which the vast majority of the tumor cells made direct contact with the surrounding 
Fig. 3 Fused Gleason pattern 4 a hematoxylin and eosin slide with subtle fibrovascular cores (arrows) and b threedimensional reconstruction revealing frequently interconnecting tubules. Cribriform Gleason pattern 4 c hematoxylin and eosin slide and d three-dimensional rendering of cribriform fields displaying contiguous epithelial cells with spherical and ellipsoid intercellular lumens. The majority of tumor cells do not contact with the surrounding stroma. The cribriform areas formed serpentine structures with variably sized interconnections (arrows). Original magnifications $20 \times$; green, Keratin 8/18 and red, Fibronectin (b) immunostaining in three-dimensional renderings
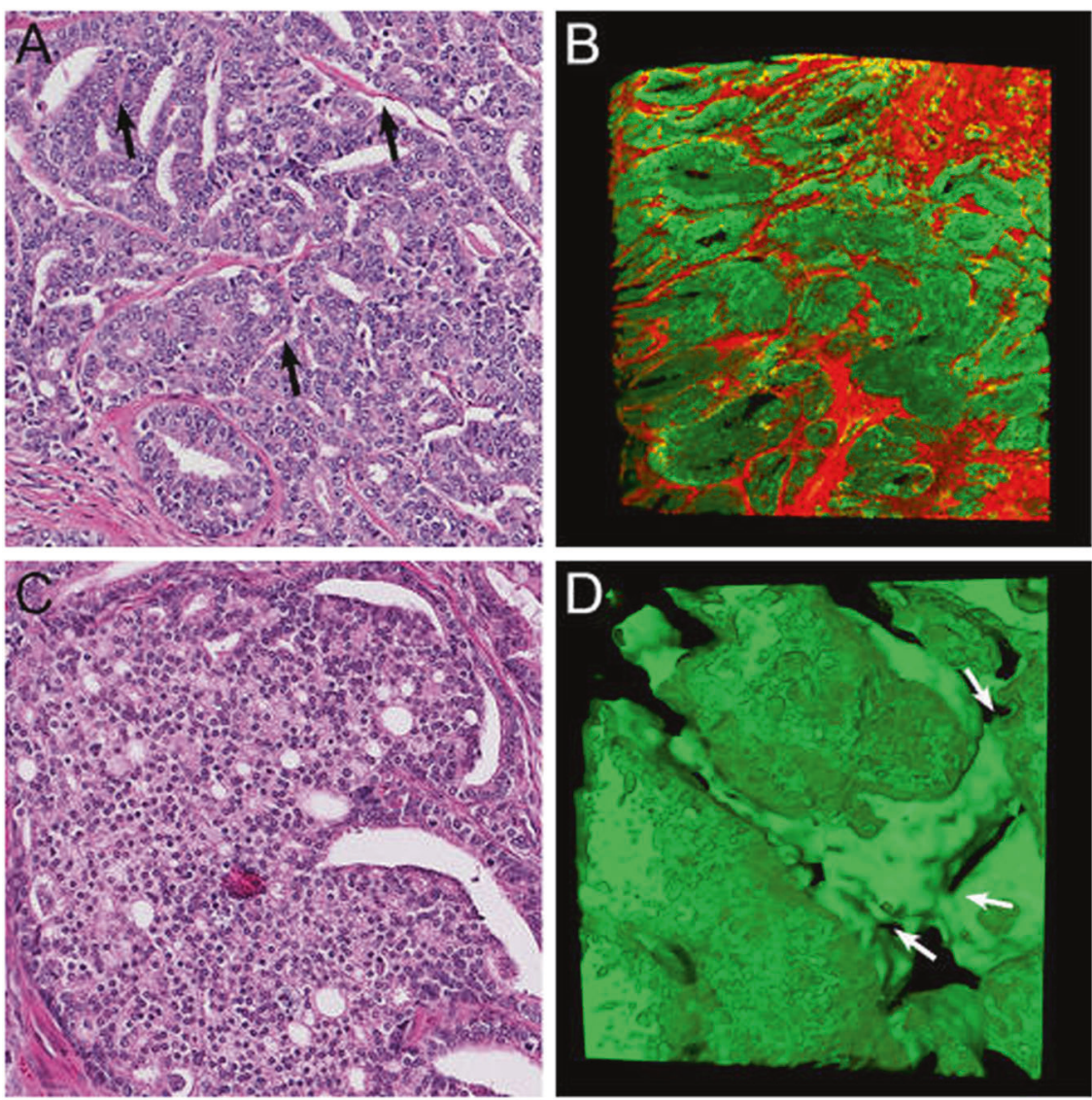

stroma. We demonstrated that Gleason pattern 3 glands formed a network of regularly interconnecting tubules. In poorly formed Gleason pattern 4, the network consisted of smaller sized tubules, and in fused Gleason pattern 4, the tubules showed frequent and closely spaced interconnections. In glomeruloid Gleason pattern 4, intraluminal epithelial protrusions occurred within the tubular network, close to tubular branching points. Cords Gleason pattern 5 represented a network structure with frequent interconnections without lumens. The second subgroup was characterized by contiguous tumor-cell proliferations in which the vast majority of tumor cells did not make any contact with the surrounding stroma, consisting of cribriform Gleason pattern 4 and solid pattern 5. This subgroup represented irregular serpentine structures of contiguous tumor cells with a decrease in frequency and size of intercellular lumens from cribriform to solid pattern.

An important advantage of three-dimensional imaging is the visualization of morphological transitions and continuity of growth patterns, which can, generally, not be appreciated in routine two-dimensional sections. Until now, only a few studies have aimed to reconstruct prostate cancer growth patterns in three dimensions, using sectioning and alignment of numerous sequential slides [15, 22-26]. For instance, Boag et al. used this method on five different cases to generate three-dimensional renderings, showing interconnections between Gleason pattern 3 and pattern 4 glands [26]. Similarly, Tolkach et al. demonstrated the continuity between Gleason pattern 3 and pattern 4 after the serial sectioning and three-dimensional rendering of one ISUP group 2 case [25]. Apart from being laborious, the stacking of sequentially cut slides is prone to tissue malformation and registration artifacts. In contrast, fluorescent staining, tissue clearing, and long-distance confocal scanning microscopy are performed on intact tissue samples without tissue sectioning, thus preventing alignment artifacts. It is also less laborious, although specialized microscopic equipment is required [19]. Our finding of the threedimensional continuity between Gleason patterns 3 and 4 is in line with the aforementioned studies, even though different methods were applied.

The most important observation of this study was that we identified two architecturally different growth pattern subgroups. Firstly, there are interconnecting tubular structures, consisting of Gleason pattern 3, poorly formed and fused Gleason pattern 4, and cords Gleason pattern 5. These patterns have variable tubule diameters, interconnection frequencies, and lumen formations, but the vast majority of the tumor cells make direct contact with the surrounding stroma. In our cohort, we observed frequent transitions 
Fig. 4 Glomeruloid Gleason pattern 4 a hematoxylin and eosin slide and $\mathbf{b}$ threedimensional rendering of small glomeruloid protrusions, consisting of malignant epithelial cells in contact with the epithelial tubular lining at one side of the tubule (arrow). c Large glomeruloid protrusions with intercellular lumens. While this structure resembles the cribriform architecture, the glomeruloid protrusion only makes contact with one side of the tubule. Original magnifications $20 \times$, green, Keratin 8/18 and red, Keratin 5 (no basal cells present) immunostaining in threedimensional renderings
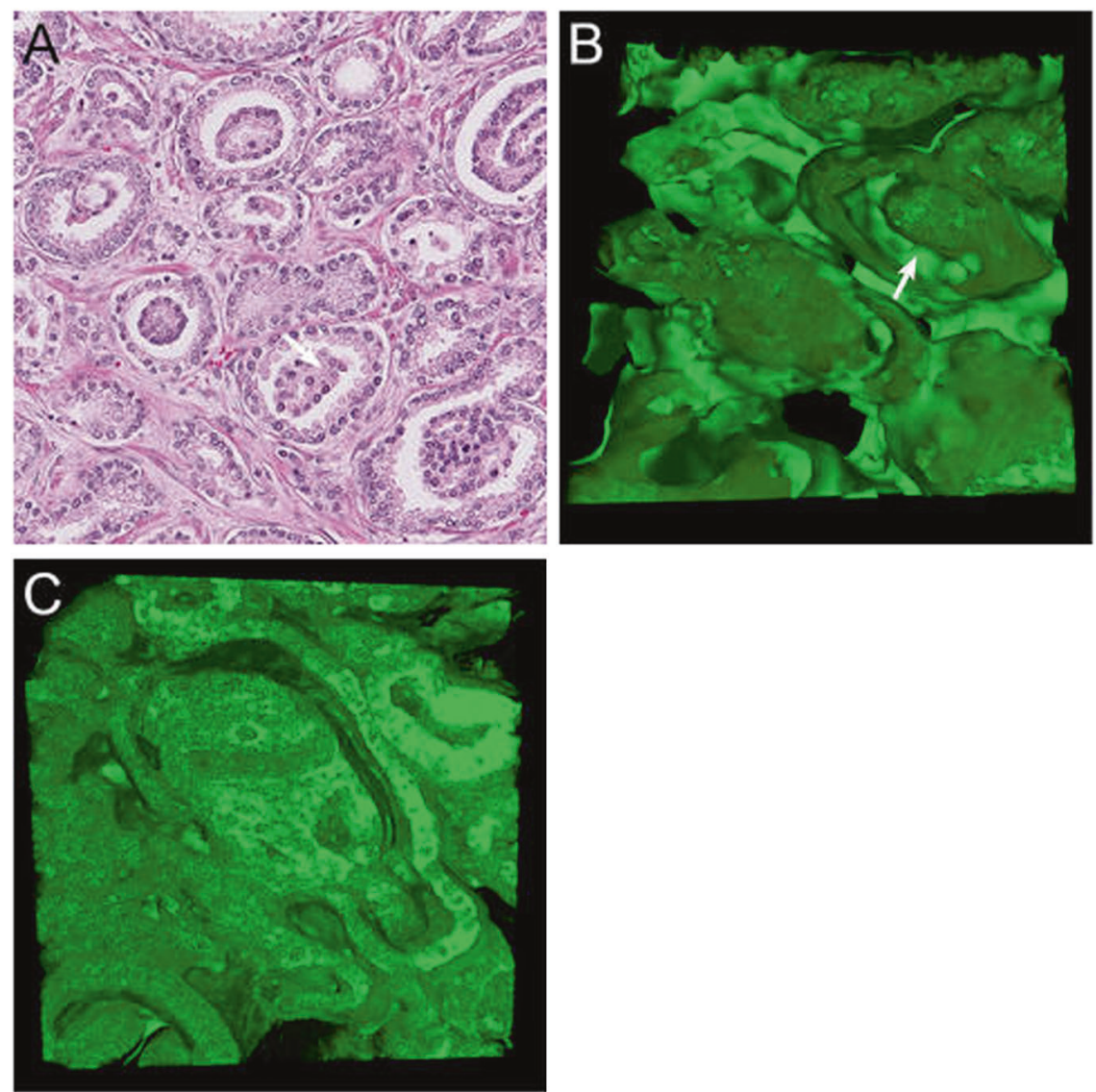

between these growth patterns. While poorly formed glands had smaller sized tubules than Gleason pattern 3, no clear cut-off could be made between these patterns with respect to tubule diameter, number of interconnections, or luminal size. Similarly, three-dimensional spatial transitions between poorly formed Gleason pattern 4 and Gleason pattern 5 cords made the strict delineation of these patterns impossible. The increased number of interconnections in fused Gleason pattern 4 was only arbitrarily distinguished from branching Gleason pattern 3 tubules. The threedimensional continuity of these patterns is reflected by the substantial inter-observer variability in daily pathology practice. Distinguishing, on one hand, tangentially sectioned Gleason pattern 3 glands from poorly formed and fused Gleason pattern 4 glands, and, on the other hand, poorly formed Gleason pattern 4 glands from Gleason pattern 5 cords on hematoxylin and eosin slides is the principal area of difficulty [12, 14, 27, 28]. Secondly, there are serpentine compact irregular epithelial proliferations, consisting of cribriform Gleason pattern 4 and solid Gleason pattern 5, with decreasing inter-epithelial lumen sizes and frequencies. Both patterns show in common that the vast majority of tumor cells are contiguous and do not make contact with the surrounding stroma. Although we did not include comedonecrosis in this study, routine diagnostic slides reveal that comedonecrosis predominantly occurs in a background of cribriform and solid structures. We found transitions between cribriform Gleason pattern 4 and solid Gleason pattern 5 but did not observe any transition between these patterns and the aforementioned tubular growth pattern subgroup.

The inter-observer agreement of cribriform Gleason pattern 4 is excellent. The only variability there occurs in the distinction between complex fused and large glomeruloid patterns [12]. Of interest, our three-dimensional images showed that although complex fused Gleason pattern 4 glands might resemble cribriform Gleason grade 4 structures on hematoxylin and eosin slides, scattered subtle intralesion fibrovascular cores were present in complex fused Gleason pattern 4 glands as a distinguishing feature. On hematoxylin and eosin slides, glomeruloid growth morphologically represents an intermediate pattern between tubular and cribriform growths. While some glomeruloid structures with subtle fibrovascular cores actually closely resembled fused Gleason pattern 4 glands in three dimensions, most glomeruloid structures did not contain intralesional connective tissue. Based on morphological resemblance and frequent coexistence, Lotan and Epstein 
Fig. 5 Gleason pattern 5 cords a hematoxylin and eosin slide and b three-dimensional rendering with interconnecting cords consisting of one or two tumor cells without lumens. Solid Gleason pattern $5 \mathbf{c}$ hematoxylin and eosin slide and $\mathbf{d}$ threedimensional rendering showing solid structures with variably sized interconnections (arrows). e Transition from cribriform Gleason pattern 4 (left side) with multiple lumens (arrows) to solid Gleason pattern 5 (right side) lacking lumen formation (arrowhead). Original magnifications $20 \times$; green, Keratin 8/18 and red, Keratin 5 (no basal cells present) immunostaining in threedimensional renderings
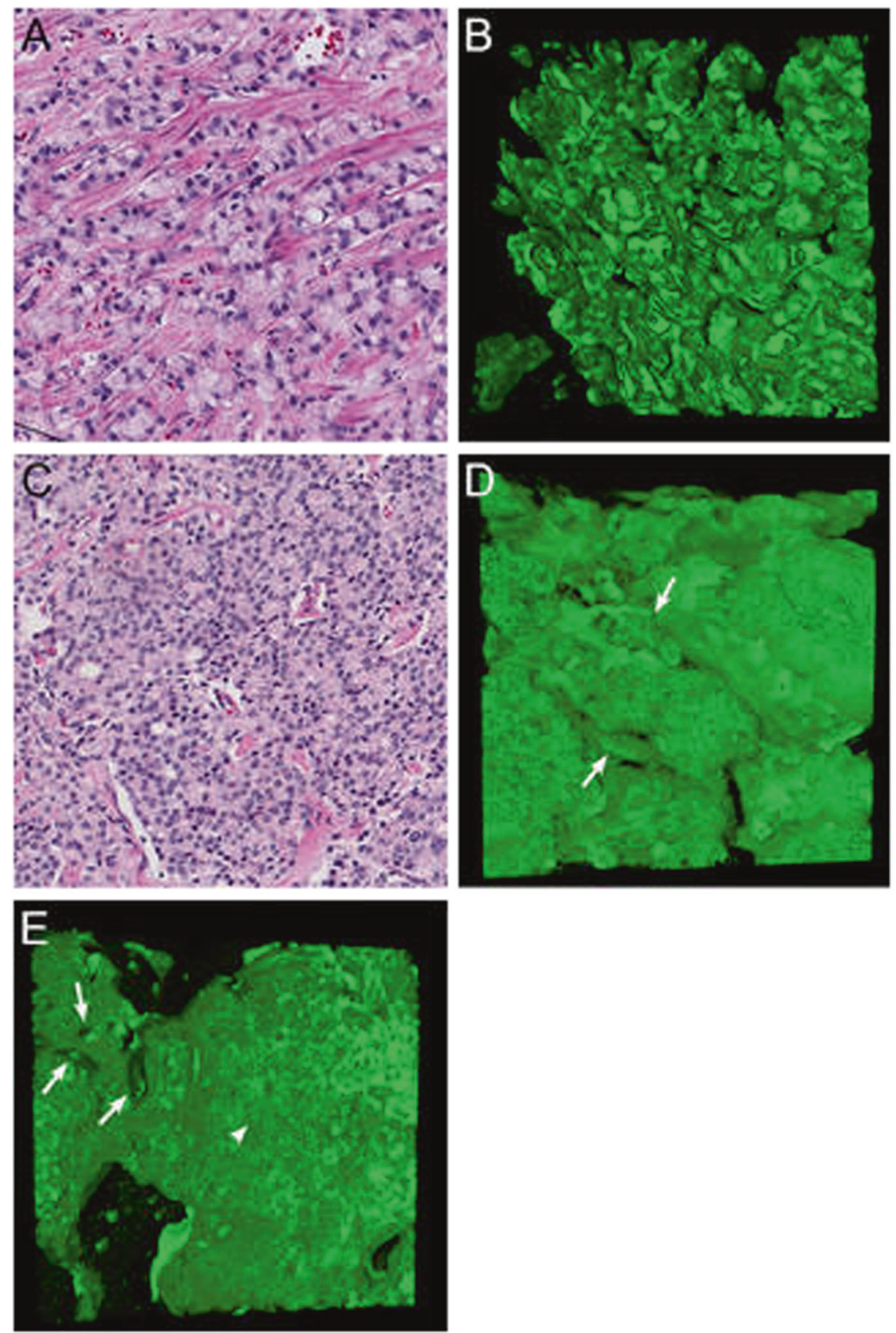

hypothesized that glomeruloid pattern is a precursor of cribriform growth [29]. However, Choy et al. found that ISUP group 2 and 3 prostate cancer patients with the glomeruloid pattern had significantly lower biochemical recurrence rates than those with cribriform growth [5]. Our three-dimensional reconstructions did not reveal any continuity between glomeruloid and cribriform structures. Therefore, the clinical relevance of the glomeruloid pattern and its place as a putative precursor of cribriform growth remain to be established.

Various studies have shown that prostate cancer patients with an ISUP group 2 tumor showing cribriform Gleason pattern 4 have a worse outcome than patients without this pattern. Kweldam et al. found that patients with an ISUP group 2 tumor without cribriform growth on biopsy had similar metastasis-free survival and biochemical recurrence rates as patients with ISUP group 1 prostate cancer [7, 8] The adverse outcome related to cribriform growth was also present in men with ISUP group $>2$ prostate cancer $[8,30]$. A putative explanation for the worse outcome of patients with cribriform growth pattern is the fact that cribriform architecture is associated with genomic instability, while non-cribriform Gleason pattern 4 is genomically indistinguishable from Gleason pattern 3 [31-33]. These clinical 
and molecular observations are in line with the two architectural subgroup hypotheses as raised in the current study. To the best of our knowledge, there are, as yet, no studies on the clinical relevance of Gleason 5 growth patterns. Investigation into the prognostic value of these individual Gleason grade 5 patterns is essential for future tumor grading and understanding. Based on our current findings, we hypothesize that solid Gleason pattern 5 is likely to be associated with a worse outcome than cords or single-cells Gleason pattern 5 .

This study represents the first comprehensive threedimensional characterization of relevant prostate cancer growth patterns. Optical clearing of intact samples allows the visualization and investigation of tumor growth patterns without the need of sectioning and alignment of numerous consecutive tissue slides. We studied the most common prostate cancer growth patterns, but did not include the full spectrum of growth patterns and variants that can be encountered in daily practice $[34,35]$. While we selected unambiguous cases of the Gleason growth patterns, interobserver variability might still exist, for instance, in labeling, as either poorly formed or fused Gleason pattern 4 $[12,13]$. Another disadvantage of three-dimensional pathology is the descriptive terminology that is used for reporting. Three-dimensional imaging of prostate cancer, however, allows for objective geometrical modeling in a three-dimensional matrix. Finally, while we identified two three-dimensional growth pattern subgroups with glomeruloid architecture as an intermediate structure, the differences between these subgroups on the clinical and molecular level remain to be investigated.

In conclusion, this study gives a comprehensive overview of the three-dimensional architecture of prostate cancer growth patterns. We show the existence of two major architectural growth pattern subgroups: (1) a tubular interconnecting network of tumor cells in direct contact with adjacent stroma, with a variable gland and lumen size, including Gleason pattern 3, poorly formed and fused Gleason pattern 4, and cords Gleason pattern 5, and (2) serpentine contiguous epithelial proliferations in which the majority of tumor cells do not make contact with the adjacent stroma and with variable inter-epithelial lumen frequency, including cribriform Gleason pattern 4 and solid Gleason pattern 5. An insight into tumor-growth patterns facilitates the comprehension of prostate cancer behavior and biology beyond the current Gleason grading.

\section{Compliance with ethical standards}

Conflict of interest The authors declare no conflict of interest.

Publisher's note: Springer Nature remains neutral with regard to jurisdictional claims in published maps and institutional affiliations.
Open Access This article is licensed under a Creative Commons Attribution 4.0 International License, which permits use, sharing, adaptation, distribution and reproduction in any medium or format, as long as you give appropriate credit to the original author(s) and the source, provide a link to the Creative Commons license, and indicate if changes were made. The images or other third party material in this article are included in the article's Creative Commons license, unless indicated otherwise in a credit line to the material. If material is not included in the article's Creative Commons license and your intended use is not permitted by statutory regulation or exceeds the permitted use, you will need to obtain permission directly from the copyright holder. To view a copy of this license, visit http://creativecommons. org/licenses/by/4.0/.

\section{References}

1. Epstein JI, Egevad L, Amin MB, Delahunt B, Srigley JR, Humphrey PA, et al. The 2014 International Society of Urological Pathology (ISUP) consensus conference on Gleason grading of prostatic carcinoma: definition of grading patterns and proposal for a new grading system. Am J Surg Pathol. 2016;40:244-52.

2. Gleason DF, Mellinger GT. Prediction of prognosis for prostatic adenocarcinoma by combined histological grading and clinical staging. J Urol. 1974;111:58-64.

3. Epstein JI. Gleason score 2-4 adenocarcinoma of the prostate on needle biopsy: a diagnosis that should not be made. Am J Surg Pathol. 2000;24:477-8.

4. Dall'Era MA, Albertsen PC, Bangma C, Carroll PR, Carter HB, Cooperberg MR, et al. Active surveillance for prostate cancer: a systematic review of the literature. Eur Urol. 2012;62:976-83.

5. Choy B, Pearce SM, Anderson BB, Shalhav AL, Zagaja G, Eggener SE, et al. Prognostic significance of percentage and architectural types of contemporary Gleason pattern 4 prostate cancer in radical prostatectomy. Am J Surg Pathol. 2016;40:1400-6.

6. Dong F, Yang P, Wang C, Wu S, Xiao Y, McDougal WS, et al. Architectural heterogeneity and cribriform pattern predict adverse clinical outcome for Gleason grade 4 prostatic adenocarcinoma. Am J Surg Pathol. 2013;37:1855-61.

7. Kweldam CF, Kummerlin IP, Nieboer D, Verhoef EI, Steyerberg $\mathrm{EW}$, Incrocci $\mathrm{L}$, et al. Prostate cancer outcomes of men with biopsy Gleason score 6 and 7 without cribriform or intraductal carcinoma. Eur J Cancer. 2016;66:26-33.

8. Kweldam CF, Kummerlin IP, Nieboer D, Verhoef EI, Steyerberg EW, van der Kwast TH, et al. Disease-specific survival of patients with invasive cribriform and intraductal prostate cancer at diagnostic biopsy. Mod Pathol. 2016;29:630-6.

9. Kweldam CF, Wildhagen MF, Steyerberg EW, Bangma CH, van der Kwast TH, van Leenders GJ. Cribriform growth is highly predictive for postoperative metastasis and disease-specific death in Gleason score 7 prostate cancer. Mod Pathol. 2015;28:457-64.

10. Al Nemer AM, Elsharkawy T, Elshawarby M, Al-Tamimi D, Kussaibi $\mathrm{H}$, Ahmed A. The updated grading system of prostate carcinoma: an inter-observer agreement study among general pathologists in an academic practice. APMIS. 2017;125:957-61.

11. Egevad L, Delahunt B, Berney DM, Bostwick DG, Cheville J, Comperat E, et al. Utility of pathology Imagebase for standardization of prostate cancer grading. Histopathology. 2018;73:8-18.

12. Kweldam CF, Nieboer D, Algaba F, Amin MB, Berney DM, Billis A, et al. Gleason grade 4 prostate adenocarcinoma patterns: an interobserver agreement study among genitourinary pathologists. Histopathology. 2016;69:441-9.

13. Egevad L, Ahmad AS, Algaba F, Berney DM, Boccon-Gibod L, Comperat E, et al. Standardization of Gleason grading among 337 European pathologists. Histopathology. 2013;62:247-56. 
14. Berney DM, Algaba F, Camparo P, Comperat E, Griffiths D, Kristiansen G, et al. The reasons behind variation in Gleason grading of prostatic biopsies: areas of agreement and misconception among 266 European pathologists. Histopathology. 2014;64:405-11.

15. Timms BG, Mohs TJ, Didio LJ. Ductal budding and branching patterns in the developing prostate. J Urol. 1994;151:1427-32.

16. Wang CW, Budiman Gosno E, Li YS. Fully automatic and robust 3D registration of serial-section microscopic images. Sci Rep. 2015;5:15051.

17. Erturk A, Lafkas D, Chalouni C. Imaging cleared intact biological systems at a cellular level by 3DISCO. J Vis Exp. 2014;89.

18. Scott GD, Blum ED, Fryer AD, Jacoby DB. Tissue optical clearing, three-dimensional imaging, and computer morphometry in whole mouse lungs and human airways. Am J Respir Cell Mol Biol. 2014;51:43-55.

19. van Royen ME, Verhoef EI, Kweldam CF, van Cappellen WA, Kremers GJ, Houtsmuller AB, et al. Three-dimensional microscopic analysis of clinical prostate specimens. Histopathology. 2016;69:985-92.

20. Renier N, Wu Z, Simon DJ, Yang J, Ariel P, Tessier-Lavigne M. iDISCO: a simple, rapid method to immunolabel large tissue samples for volume imaging. Cell. 2014;159:896-910.

21. Schindelin J, Arganda-Carreras I, Frise E, Kaynig V, Longair M, Pietzsch T, et al. Fiji: an open-source platform for biologicalimage analysis. Nat Methods. 2012;9:676-82.

22. Kay PA, Robb RA, Bostwick DG. Prostate cancer microvessels: a novel method for three-dimensional reconstruction and analysis. Prostate. 1998;37:270-7.

23. Gibson E, Gaed M, Gomez JA, Moussa M, Pautler S, Chin JL, et al. 3D prostate histology image reconstruction: Quantifying the impact of tissue deformation and histology section location. J Pathol Inform. 2013;4:31.

24. Singh M, Kalaw EM, Giron DM, Chong KT, Tan CL, Lee HK. Gland segmentation in prostate histopathological images. J Med Imaging (Bellingham). 2017;4:027501.

25. Tolkach Y, Thomann S, Kristiansen G. 3D-reconstruction of prostate cancer architecture with serial immunohistochemical sections: hallmarks of tumour growth, tumour compartmentalization and implications for grading and heterogeneity. Histopathology. 2018;72:1051-9.

26. Boag AH, Kennedy LA, Miller MJ. Three-dimensional microscopic image reconstruction of prostatic adenocarcinoma. Arch Pathol Lab Med. 2001;125:562-6.

27. Shah RB, Li J, Cheng L, Egevad L, Deng FM, Fine SW, et al. Diagnosis of Gleason pattern 5 prostate adenocarcinoma on core needle biopsy: an interobserver reproducibility study among urologic pathologists. Am J Surg Pathol. 2015; 39:1242-9.

28. Zhou M, Li J, Cheng L, Egevad L, Deng FM, Kunju LP, et al. Diagnosis of "poorly formed glands" Gleason pattern 4 prostatic adenocarcinoma on needle biopsy: an interobserver reproducibility study among urologic pathologists with recommendations. Am J Surg Pathol. 2015;39:1331-9.

29. Lotan TL, Epstein JI. Gleason grading of prostatic adenocarcinoma with glomeruloid features on needle biopsy. Hum Pathol. 2009;40:471-7.

30. Hannezo E, Scheele C, Moad M, Drogo N, Heer R, Sampogna $\mathrm{RV}$, et al. A unifying theory of branching morphogenesis. Cell. 2017;171:242-55. e27

31. Bottcher R, Kweldam CF, Livingstone J, Lalonde E, Yamaguchi $\mathrm{TN}$, Huang V, et al. Cribriform and intraductal prostate cancer are associated with increased genomic instability and distinct genomic alterations. BMC Cancer. 2018;18:8.

32. Chua MLK, Lo W, Pintilie M, Murgic J, Lalonde E, Bhandari V, et al. A prostate cancer "nimbosus": genomic instability and SChLAP1 dysregulation underpin aggression of intraductal and cribriform subpathologies. Eur Urol. 2017;72:665-74.

33. Williams JL, Greer PA, Squire JA. Recurrent copy number alterations in prostate cancer: an in silico meta-analysis of publicly available genomic data. Cancer Genet. 2014;207:474-88.

34. Humphrey PA. Variants of acinar adenocarcinoma of the prostate mimicking benign conditions. Mod Pathol. 2018;31:S64-70.

35. McKenney JK, Wei W, Hawley S, Auman H, Newcomb LF, Boyer $\mathrm{HD}$, et al. Histologic grading of prostatic adenocarcinoma can be further optimized: analysis of the relative prognostic strength of individual architectural patterns in 1275 patients from the Canary Retrospective Cohort. Am J Surg Pathol. 2016;40:1439-56. 